\title{
The Unified Theory of Resonance Energy Transfer According to Molecular Quantum Electrodynamics
}

\section{A. Salam}

Department of Chemistry, Wake Forest University, Winston-Salem, NC 27109-7486, USA; salama@wfu.edu; Tel.: +1-336-758-3713

Received: 10 September 2018; Accepted: 8 October 2018; Published: 11 October 2018

\begin{abstract}
An overview is given of the molecular quantum electrodynamical (QED) theory of resonance energy transfer (RET). In this quantized radiation field description, RET arises from the exchange of a single virtual photon between excited donor and unexcited acceptor species. Diagrammatic time-dependent perturbation theory is employed to calculate the transfer matrix element, from which the migration rate is obtained via the Fermi golden rule. Rate formulae for oriented and isotropic systems hold for all pair separation distances, $R$, beyond wave function overlap. The two well-known mechanisms associated with migration of energy, namely the $R^{-6}$ radiationless transfer rate due to Förster and the $R^{-2}$ radiative exchange, correspond to near- and far-zone asymptotes of the general result. Discriminatory pair transfer rates are also presented. The influence of an environment is accounted for by invoking the polariton, which mediates exchange and by introducing a complex refractive index to describe local field and screening effects. This macroscopic treatment is compared and contrasted with a microscopic analysis in which the role of a neutral, polarizable and passive third-particle in mediating transfer of energy is considered. Three possible coupling mechanisms arise, each requiring summation over 24 time-ordered diagrams at fourth-order of perturbation theory with the total rate being a sum of two- and various three-body terms.
\end{abstract}

Keywords: molecular quantum electrodynamics; resonance energy transfer; virtual photon exchange; Förster transfer; radiative exchange; discriminatory transfer; medium effects; polariton mediated exchange

\section{Introduction}

A wide-ranging fundamental process is the transfer of energy between matter [1]. A particularly interesting special case is the resonant migration of excitation energy [2], which frequently takes place between atoms, molecules, chromophores, functional groups or other such chemical units. Electronic and/or vibrational energy is localized on one initially pre-excited species that subsequently decays, often to the ground state and transfers its energy resonantly to a second entity, which is initially in its lowest energy level but which becomes excited on acquisition of all of the energy exchanged. There are clearly spatio-temporal aspects that feature in the transfer process and which must be accounted for regardless of whether the exchange of energy is resonant or not since the donor and acceptor moieties are separated from one another in both the space and time dimensions.

The intrinsic importance of the problem of resonance energy transfer (RET) is evident from its formulation, as stated above and the fact that it manifests in an inordinately large number of varied scientific and engineering situations and applications ranging from revealing structural information on biomolecules, to improving the functionality and efficiency of devices fabricated at the nanoscale [3-9]. A considerable amount of theoretical work has been carried out over the years in order to understand the basic mechanism by which energy is transferred in biological, chemical and physical systems. Here we give an overview of the application of the theory of molecular quantum electrodynamics 
(QED) to RET, concentrating first on the pair transfer rate before going on to examine the role of an additional microscopic particle or a medium on the exchange process.

\section{Early Theoretical Studies}

The study of RET, especially within the chemical context, goes back to the early days of the application of quantum theory, which arose from the strong desire to understand the forces between atoms and molecules in excited electronic states, the origin of resonance forms and the stability of molecular bonds $[10,11]$. Soon after the non-relativistic quantum mechanical theory of particles was finalized, attention shifted to developing a quantum theory of electromagnetic radiation and which led to early theories of quantum electrodynamics [12-15]. This was motivated in large part by developments in atomic and molecular spectroscopy. These advances in fundamental theory permitted more rigorous investigations of the RET problem to be undertaken. An important early study was carried out by Fermi [15] in which many of the key physical issues embodied in the problem were delineated and tackled. At the same time, the RET process was cast in the broadest of terms possible, thereby enabling it to be viewed, for instance, as the exchange or transfer of energy between emitter and absorber, transmitter and receiver, antenna and detector, among others, in addition to the chemical scenarios already mentioned, in an early recognition of the diverse situations in which RET manifests.

Arguably the most far-reaching feature considered by Fermi is that associated with signal propagation. With the decay of excitation energy by the donor being accompanied by emission of electromagnetic radiation, obviously the acceptor cannot absorb this radiation at a time earlier than $R / c$, where $R$ is the distance of the receiver from the emitter and $c$ is the speed of light. If this were to occur, then strict Einstein causality would be violated, which is patently unphysical. Fermi calculated the quantum mechanical probability for energy to be transferred between the pair resonantly by using time-dependent perturbation theory and found that certain approximations had to be made in the computation otherwise acausal transfer of energy would ensue. This entailed discarding non-resonant terms and when performing the integral over frequency of the remaining contributions, extending the limits from zero to infinity to minus infinity to infinity. The inadequacy of this analysis has prompted numerous studies over the years looking into this aspect of the problem [16-32], shedding considerable light onto issues surrounding fundamental tests of the validity of quantum mechanics and measurement theory arising from implications associated with the correct state specification of such a system. These have now largely been resolved [32]. The consensus being that to arrive at a solution that preserves causality is to calculate, without any mathematical approximations within time-dependent perturbation theory, the probability that the receiver detects a quantum of excitation energy without any reference to the state of the emitter or to that of the radiation field. Such a treatment is necessary if the particles exchanging energy are non-identical but with the proviso that the species have overlapping energy spectra. The picture is therefore one in which the transfer of energy is irreversible. Additional understanding has been gained from the study of the closely related problem of the van der Waals dispersion energy shift between one ground and one excited atom or molecule [33-37].

In addition to the interest associated with preparing and measuring particular system states and the temporal evolution of the RET probability, a second, equally important aspect that motivated much effort surrounded the actual physical mechanism underlying the process. In this regard, pioneering work was carried out by Förster [17]. He took the electric dipole moments associated with the excited atom and the ground state one to be coupled by the static dipolar interaction

$$
V_{i j}^{\text {static }}=\frac{1}{4 \pi \varepsilon_{0} R^{3}}\left(\delta_{i j}-3 \hat{R}_{i} \hat{R}_{j}\right),
$$

where $R$ is the displacement of the acceptor with respect to the donor. The Latin subscripts denote Cartesian tensor components. Application of second-order perturbation theory led to a quantum mechanical probability and a transfer rate that displayed inverse sixth power separation distance 
dependence, the process named after Förster. Because the coupling between centers Equation (1) is instantaneous in its action, migration of excitation energy is referred to as being radiationless. The rate formula applies to short distances outside the overlap region of molecular charge distributions. Within the overlap region, much stronger exchange and repulsion interactions operate, with early studies carried out by Dexter within the context of sensitized fluorescence [38]. We shall not be concerned with these second types of forces concentrating instead on descriptions of transfer beyond wave function overlap [1,4,39-41]. Three distance regimes may therefore be identified: (i) separations within the overlap region of wave functions, which may be termed ultra-short range; (ii) distances over which the coupling between centers is non-retarded, which may be called short range; and (iii) displacements at which retardation effects dominate, which are referred to as long range.

If migration of energy is viewed as being transmitted by electromagnetic fields as a result of emission of radiation by the excited atom followed by its absorption by the ground state species, as intimated earlier, then outside overlap and beyond the region at which the $R^{-6}$ form of rate law would be expected to hold, namely at long range, transfer should occur by a mechanism that is radiative in origin. A theoretical framework that rigorously describes light propagation is therefore necessary.

\section{Scope}

Such a theory is quantum electrodynamics (QED) [42,43], which will be shown to yield a unified model for RET [44-48], correctly describing behavior at short and long ranges, as well as in the intermediate zone that lies in between the extremes of the non-retarded and retarded regimes. An advantage in using QED is that the electromagnetic field is an intrinsic part of the system. Because matter and radiation are subject to quantum mechanical laws, light quanta-the photons, are accounted for automatically by the theory. This means that QED may be readily applied to treat the interaction of electromagnetic fields with matter, as well as to the interaction between matter. In the case of atoms and molecules, a non-relativistic formulation of QED theory has dealt very successfully with problems occurring in the areas of theoretical spectroscopy and forces between microscopic particles [49-52]. It will be shown that in the perturbation theory approach to the computation of the probability amplitude, RET is understood to arise from the exchange of a single virtual photon between the coupled pair $[20,47,53-55]$. Such photons are by definition undetectable but as is characteristic of quantum field theories, mediate the interaction between material particles. In a similar vein, the van der Waals dispersion potential between a pair of neutral atoms or molecules in the ground electronic state is interpreted as arising from two virtual photon exchanges $[49-51,56,57]$. In what follows emphasis will be placed on the molecular QED theory of RET. Applications will therefore be downplayed, with the focus instead directed at the basic equations and their physical origin. Hence with this objective in mind, only key publications will be cited.

The presentation is organized as follows. In the next Section a brief introduction to the molecular QED formalism and diagrammatic perturbation theory is given. This will be sufficient to enable the general solution to the problem of RET to be obtained. Theory is applied in Sections 5 and 6 to RET occurring between a pair of electric dipole species. Diagrammatic perturbation theory is used to evaluate the transfer matrix element, from which the probability and rate are arrived at via the Fermi golden rule. The resulting expressions apply to particles in fixed mutual orientation, as well as to freely tumbling species, for all separation distances beyond the contact distance. Asymptotically limiting forms of the rate, which hold at short and long range, are extracted from the general formula and shown to correspond to the radiationless and radiative transfer processes, respectively, as described above. In Section 7 the effect that an environment has in modifying the RET rate is examined. This is done through a description in terms of polaritons, which alters the form of the coupling tensor by taking into account the refractive index of the medium. Next the electric dipole approximation is relaxed and the effects of higher multipole moment terms are considered. Of special interest in Section 8 is the contribution to the transfer rate that is dependent upon the transition electric and magnetic dipole moment of each center. Such a term necessarily describes the chirality of the particular species 
and leads to transfer rates that are discriminatory, that is, dependent upon the handedness of the individual molecules. In Section 9 the pair theory is extended to include the effect that a passive neutral polarizable molecule has in mediating transfer of resonant excitation energy between donor and acceptor units. While the preferred role of the third body, from the viewpoint of transfer efficiency, is to serve as a bridging species, two other pathways are possible in which the third particle couples only to the donor moiety, or only to the acceptor unit. These are considered too. Not only are there contributions to the rate arising from each of these three routes but the total rate contains interference terms between direct (two-body) and indirect (third-body mediated) transfer, as well as interference terms between the different three-body mechanisms. Finally, in Section 10, a brief summary is given along with an outline of possible future prospects.

\section{Stationary State Approximation}

A commonly held view of resonant transfer of excitation energy between identical species A and $B$ is that afforded by secular perturbation theory at second order in which electronic and/or vibrational energy is localized at either center in the stationary state approximation. In this model, the wave function is given by the symmetric and anti-symmetric linear combination

$$
\left|\Psi_{ \pm}\right\rangle=\frac{1}{\sqrt{2}}\left(\left|m^{\mathrm{A}}, 0^{\mathrm{B}}\right\rangle \pm\left|0^{\mathrm{A}}, m^{\mathrm{B}}\right\rangle\right)
$$

for excitation from the ground state $|0\rangle$ to an excited state $|m\rangle$ and which suggests that the coupled pair are in fairly close proximity to one another. Such a description holds when the exchange of energy is rapid relative to decay via fluorescence or collisional deactivation processes but it results in no overall exchange of excitation energy. De-excitation occurs via emission by the coupled pair, leading to the well-known results that when the transition dipole moments in the two species are parallel to one another, application of selection rules produces a rate that is close to twice that for a single molecule for decay from the symmetric state, denoted by the "+" sub-script in Equation (2) and which vanishes for a transition from $\left|\Psi_{-}\right\rangle$. The approximation breaks down, however, when it is impossible to detect one or the other of the two stationary states Equation (2), or for larger separations. Then it becomes necessary to adopt a time-dependent formulation of the problem. And for a treatment based on QED theory, this additionally involves accounting for the interaction of each species with the quantized electromagnetic field. Inclusion of both of these aspects from the start is therefore essential to obtain a rigorous solution to and for a fundamental understanding of, the energy migration process. This is examined next.

\section{Molecular QED Theory and Diagrammatic Perturbation Theory}

The RET matrix element, taking explicit account of the dynamic nature of the exchange, may be obtained within the framework of non-relativistic QED theory, in which atoms and molecules, containing bound electrons, move with speeds that are a tiny fraction of that of light. The calculation is carried out using the standard techniques of time-dependent perturbation theory in conjunction with associated Feynman like diagrams depicting all of the possible time-ordered sequences of photon annihilation and creation events that connect the same initial and final states that describe the process in question. For donor particle D positioned at $\vec{R}_{\mathrm{D}}$, acceptor moiety A located at $\vec{R}_{\mathrm{A}}$, with pair separation distance $\vec{R}=\vec{R}_{\mathrm{A}}-\vec{R}_{\mathrm{D}}$, the total QED Hamiltonian operator for the particle-field system is

$$
H=H_{\mathrm{mol}}(\mathrm{D})+H_{\mathrm{mol}}(\mathrm{A})+H_{\mathrm{rad}}+H_{\mathrm{int}}(\mathrm{D})+H_{\mathrm{int}}(\mathrm{A}) .
$$

As is characteristic of QED, the electromagnetic field is an intrinsic part of the quantum mechanical system and its Hamiltonian operator features explicitly in Equation (3), representing the total energy of the free radiation field. It may be interpreted as a collection of non-interacting simple harmonic 
oscillators, which on quantization results in the photon, the vibrations of the electromagnetic field being described unambiguously by the propagation vector $\vec{k}$, and polarization characteristics, $\lambda$. Adopting the second quantization scheme through the introduction of boson annihilation and creation operators for a $(\vec{k}, \lambda)$ - mode photon, $a^{(\lambda)}(\vec{k})$ and $a^{\dagger(\lambda)}(\vec{k})$, respectively, facilitates expression of $H_{\text {rad }}$ as

$$
H_{\mathrm{rad}}=\sum_{\vec{k}, \lambda}\left\{a^{\dagger(\lambda)}(\vec{k}) a^{(\lambda)}(\vec{k})+\frac{1}{2}\right\} \hbar \omega
$$

where the circular frequency, $\omega=c|\vec{k}|$. In this representation, the operator combination $a^{\dagger(\lambda)}(\vec{k}) a^{(\lambda)}(\vec{k})$ is interpreted as the number operator, $n(\vec{k}, \lambda)$, whose eigenvalues are the number of light quanta of mode $(\vec{k}, \lambda)$, with energy $n \hbar \omega$, via the relation

$$
n(\vec{k}, \lambda)|n(\vec{k}, \lambda)\rangle=a^{\dagger(\lambda)}(\vec{k}) a^{(\lambda)}(\vec{k})|n(\vec{k}, \lambda)\rangle
$$

where the eigenstates of the radiation field are the number states $|n(\vec{k}, \lambda)\rangle \cdot a^{(\lambda)}(\vec{k})$ and $a^{\dagger(\lambda)}(\vec{k})$ respectively decrease or increase by one the number of photons in the radiation field. It is interesting to note that the ground state of the electromagnetic field is the one in which there is a complete absence of photons of any and all modes, represented by the ket

$$
\left|0\left(\vec{k}_{1}, \lambda_{1}\right), 0\left(\vec{k}_{2}, \lambda_{2}\right), \ldots, 0\left(\vec{k}_{i}, \lambda_{i}\right), \ldots\right\rangle \text {. }
$$

This corresponds to the electromagnetic vacuum state. Despite the lack of photons, the field possesses a non-vanishing energy of $\frac{1}{2} \hbar \omega$ per mode and is known as the zero-point energy per photon. Returning to Equation (3), the first two terms denote the molecular Hamiltonians of the donor and acceptor species. Their detailed forms are familiar from molecular quantum mechanics in the Born-Oppenheimer approximation, comprising a sum of kinetic and intra-molecular potential energy terms.

Collectively, the last two terms of Equation (3) represent the coupling operator. In the widely used multipolar form of molecular QED theory $[43,49,50]$, atoms and molecules interact directly with the electromagnetic field through their multipole moment distributions, comprising electric polarization, magnetization and diamagnetization components. Coupling between matter takes place via the exchange of one or more virtual photons, emitted at one center and absorbed at another site. Theory permits both real and virtual photons to occur, with the latter providing a useful means for allowing interactions between particles to be calculated. In the case of RET, the interaction arises from the exchange of a single virtual photon between the coupled donor-acceptor pair. On retaining the leading term in the expansion of the multipolar series, corresponding to electric dipole coupling, the interaction Hamiltonian is written as

$$
H_{\mathrm{int}}(\mathrm{D})+H_{\mathrm{int}}(\mathrm{A})=-\varepsilon_{0}^{-1} \vec{\mu}(\mathrm{D}) \cdot \vec{d}^{\perp}\left(\vec{R}_{\mathrm{D}}\right)-\varepsilon_{0}^{-1} \vec{\mu}(\mathrm{A}) \cdot \vec{d}^{\perp}\left(\vec{R}_{\mathrm{A}}\right),
$$

where $\vec{\mu}$ is the electric dipole moment operator. In the multipolar version of the theory, $\vec{\mu}$ couples to the transverse electric displacement field operator, $\vec{d}^{\perp}(\vec{r})$. In terms of Fourier components, its mode expansion is given by

$$
\vec{d}^{\perp}(\vec{r})=i \sum_{\vec{k}, \lambda}\left(\frac{\hbar c k \varepsilon_{0}}{2 V}\right)^{1 / 2}\left[\vec{e}^{(\lambda)}(\vec{k}) a^{(\lambda)}(\vec{k}) \mathrm{e}^{i \vec{k} \cdot \vec{r}}-\overrightarrow{\vec{e}}^{(\lambda)}(\vec{k}) a^{\dagger(\lambda)}(\vec{k}) \mathrm{e}^{-i \vec{k} \cdot \vec{r}}\right]
$$


where $\vec{e}^{(\lambda)}(\vec{k})$ is the complex unit electric polarization vector, the overbar denotes the complex-conjugate quantity and $V$ is the box quantization volume, invoked to restrict the number of allowed modes to a countable infinity. The collection of quantities occurring within parentheses is a normalization factor, ensuring that the field contributes correctly to the energy of the electromagnetic radiation for any specific state of the field.

The molecular Hamiltonians and the radiation field Hamiltonian constitute the unperturbed Hamiltonian operator, whose base states are taken to be product molecule-radiation field states since in the absence of interaction, $H_{\mathrm{mol}}$ and $H_{\mathrm{rad}}$ are separable. The effect of the interaction Hamiltonian is to cause transitions between initial and final states of the system, with the probability amplitude to a given order in $H_{\text {int }}$ calculated via time-dependent perturbation theory.

From the statement of the RET problem, it is a simple matter to write down the total system initial and final states. They are:

$$
|i\rangle=\left|m^{\mathrm{D}}, 0^{\mathrm{A}}\right\rangle,
$$

and

$$
|f\rangle=\left|0^{\mathrm{D}}, m^{\mathrm{A}}\right\rangle,
$$

where $\left|0^{\xi}\right\rangle$ and $\left|m^{\xi}\right\rangle$ designate ground and $m$-th excited electronic states of species $\xi$, respectively. Because there are no photons present initially or finally, the state of the radiation field has been omitted from the temporal system specifications. On account of RET occurring via single virtual photon exchange to leading order, the probability amplitude is evaluated perturbatively by employing the formula for the second-order correction to the interaction Hamiltonian,

$$
M_{f i}^{(2)}=-\sum_{I} \frac{\left\langle f\left|H_{\text {int }}\right| I\right\rangle\left\langle I\left|H_{\text {int }}\right| i\right\rangle}{E_{I i}}
$$

where the sum is executed over all intermediate radiation-molecule states that connect $|i\rangle$ to $|f\rangle$. Appearing in the denominator of Expression (10) is the difference between the intermediate and initial state energies, $E_{I}-E_{i}$, simplified notationally to $E_{I i}$.

Time-ordered diagrams provide a convenient graphical counterpart to the algebraic form of the time-dependent perturbation theory expansion of the matrix element. These Feynman like diagrams depict individual photon-matter coupling events [58-60]. All topologically distinct time-ordered sequences that link the same initial and final states at a given order of perturbation theory contribute to the probability amplitude, with a particular diagram representing one term in the perturbative sum over intermediate states. For higher-order processes, or for those involving many-centers, in which the number of time-orderings can become very large, one diagram, called a state-sequence diagram, may be used to represent all of the possible time-ordered sequences and provides an advantageous alternative to the usual Feynman-like depiction. Details on how they are constructed and deployed in simple applications may be found in Ref. [61].

In the case of RET, since virtual emission followed by absorption may occur at either site, two distinct time-ordered diagrams may be drawn, the sum of whose amplitudes yields the correct total matrix element at second-order in the coupling [54]. The two graphs are shown in Figure 1. In these variants to Feynman diagrams, applicable to light interacting with non-relativistic charged particles, time progresses upwards with a solid line depicting the state of the atom or molecule. An angled dashed line indicates a photon, characterized by its mode. Intersection of dashed and solid lines corresponds to radiation-matter coupling. In the present example, the electric dipoles of D and A interact with the electric displacement field, with the coupling Hamiltonian given by Equation (7). The mode of the single virtual photon exchanged between the pair is labelled $(\vec{p}, \varepsilon)$. The polarization is summed over all index values and integration is performed over all possible values of the momentum 
of the virtual photon. The conversion of discrete wave vectors to a continuous variable is done through the relation

$$
\lim _{V \rightarrow \infty} \frac{1}{V} \sum_{\vec{p}} \rightarrow \int_{0}^{\infty} \frac{d^{3} \vec{p}}{(2 \pi)^{3}}
$$

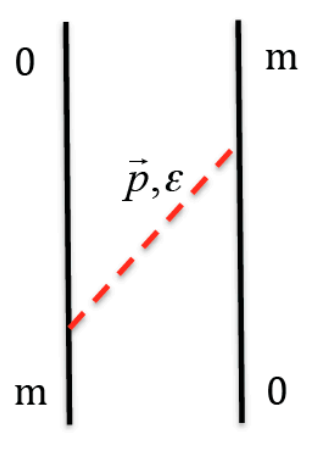

D A

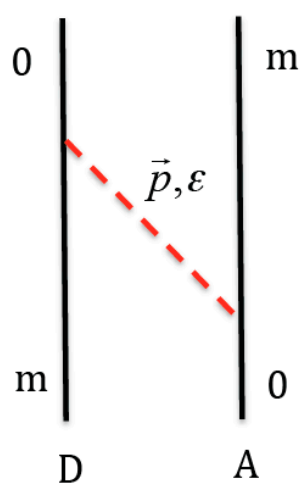

Figure 1. The two time-ordered diagrams depicting pair resonance energy transfer.

In a similar vein, a sum over all virtual levels accessible by electric dipole allowed transitions in either species is required. Both of these aspects arise automatically in the time-dependent perturbation theory treatment when evaluating the sum over all possible intermediate states associated with matter and field.

From Figure 1 it is seen that the diagram on the left gives rise to an intermediate state in which both particles are in the ground state and one virtual photon is in transit, while the other diagram produces the intermediate state in which D and A are both electronically excited in state $|m\rangle$, with one virtual photon again in propagation, this time emanating from center A. Utilizing the initial, intermediate and final states, together with the perturbation operator Equation (7) in Formula (10) yields for the sum of both diagrams in Figure 1, the probability amplitude

$$
M_{f i}^{(2)}=-\frac{1}{4 \pi^{2} \varepsilon_{0}} \mu_{i}^{0 m}(\mathrm{D}) \mu_{j}^{m 0}(\mathrm{~A})\left(-\nabla^{2} \delta_{i j}+\nabla_{i} \nabla_{j}\right) \frac{1}{R} \int_{0}^{\infty} \sin p R\left\{\frac{1}{p-k_{m 0}}+\frac{1}{p+k_{m 0}}\right\} d p,
$$

where $R=\left|\vec{R}_{\mathrm{A}}-\vec{R}_{\mathrm{D}}\right|$ is the pair separation distance. An implied Einstein summation convention has been adopted for repeating Cartesian tensor component indices. How the integral over virtual photon momentum has been carried out and the form of the resulting matrix element, have been the subject of intense scrutiny over the years [20,21,28,46,53].

First, it is important to realize that the second-order expression for the probability amplitude Equation (12) has been obtained under the assumption that no intermediate state resonances occur. For migration of energy that is near-resonant, the amplitude is found to not vanish for $R>c t$ [28]. To ensure a result that is in accord with strict Einstein causality, all exponential terms with time-dependent arguments must be retained. It is found that the physically acceptable matrix element is obtained by taking the Cauchy principal value in the integration since the contributions from poles mutually cancel. Prior to this exact evaluation, a commonly made simplifying procedure is the rotating-wave approximation, in which counter-rotating terms are discarded. A causal result follows on extending the lower limit of the wave vector integral from 0 to $-\infty$. This was Fermi's original solution to the RET problem [15]. It is found to agree with the matrix element obtained by coupling the electric dipole of one of the species to the causal driving field due to the other species that is considered as a source of electromagnetic radiation as it undergoes a downward electric dipole allowed transition with frequency $\omega_{m 0}=E_{m 0} / \hbar[24,62]$. 
A second important factor that bears on the issue at hand is whether transfer is reversible or irreversible. If the former, the commonly employed stationary state approximation, in which there is no net transfer of energy, produces for the integral in Equation (12) the result $-\pi \cos k_{m 0} R$, which is equivalent to taking the Cauchy principal value. An oscillatory behavior for exchange of energy ensues. If the Fermi golden rule is used to then compute a transfer rate, this necessarily implies that migration of energy is unidirectional and therefore irreversible. In such a case time-dependent perturbation theory yields the complex exponential form on evaluation of the wave vector integral. Since we will be concerned with an excitation transfer rate, evaluation of Equation (12) produces for the matrix element,

$$
M_{f i}^{(2)}=-\mu_{i}^{0 m}(\mathrm{D}) \mu_{j}^{m 0}(\mathrm{~A})\left(-\nabla^{2} \delta_{i j}+\nabla_{i} \nabla_{j}\right) \frac{e^{i k_{m 0} R}}{4 \pi \varepsilon_{0} R} .
$$

Implicit within the form of result Equation (13) is the retarded dipole-dipole coupling tensor, which is defined as

$$
\begin{aligned}
& V_{i j}(k, \vec{r})=-\frac{1}{4 \pi \varepsilon_{0}}\left(-\nabla^{2} \delta_{i j}+\nabla_{i} \nabla_{j}\right) \frac{e^{i k r}}{r} \\
& =\frac{1}{4 \pi \varepsilon_{0} r^{3}}\left[\left(\delta_{i j}-3 \hat{r}_{i} \hat{r}_{j}\right)(1-i k r)-\left(\delta_{i j}-\hat{r}_{i} \hat{r}_{j}\right) k^{2} r^{2}\right] \mathrm{e}^{i k r},
\end{aligned}
$$

enabling the matrix element Equation (13) to be written as

$$
M_{f i}^{(2)}=\mu_{i}^{0 m}(\mathrm{D}) \mu_{j}^{m 0}(\mathrm{~A}) V_{i j}\left(k_{m 0}, \vec{R}\right)
$$

To avoid ambiguities in the choice of contour and the displacement of poles when carrying out the wave vector integral in the complex frequency plane, special functions have been employed to arrive at the matrix element [46]. Additional valuable insight has been gained into the problem of RET [48] by describing the exchanged virtual photon as a spherical rather than as a plane wave. As a consequence of this, the identification of incoming and outgoing wave conditions arises automatically. Informative studies into the nature of the electronic coupling at the Förster limit compared with the full QED matrix element and their distance dependence have been carried out by simulating the exciton dynamics associated with an array of dipoles arranged in a brickstone lattice, confirming the breakdown of the near-zone approximation as the distance between dipoles increases [63].

\section{Transfer Rate}

Within this weak coupling regime, the transfer rate may be evaluated via the Fermi golden rule,

$$
\Gamma=\frac{2 \pi}{\hbar}\left|M_{f i}\right|^{2} \rho_{f}
$$

where $\rho_{f}$ is the density of final states. The concept of a rate holds for timescales lying within the range $\omega_{m 0}^{-1}<<t<<\Gamma^{-1}$, where $\omega_{m 0}$ is the resonant transition frequency. Inserting Equation (15) into (16) gives an expression for the rate of RET applicable to D and A in fixed mutual orientation,

$$
\Gamma=\frac{2 \pi}{\hbar} \rho_{f} \mu_{i}^{0 m}(\mathrm{D}) \bar{\mu}_{i^{\prime}}^{m 0}(\mathrm{D}) \mu_{j}^{m 0}(\mathrm{~A}) \bar{\mu}_{j^{\prime}}^{0 m}(\mathrm{~A}) V_{i j}\left(k_{m 0}, \vec{R}\right) \bar{V}_{i^{\prime} j^{\prime}}\left(k_{m 0}, \vec{R}\right) .
$$

When interest is confined to migration of energy between species in the fluid phase, a rotational average [64] is taken of Equation (17). Multiplying the interaction tensors and contracting with $\delta_{i i^{\prime}} \delta_{j j^{\prime}}$ arising from orientational averages taken over the transition electric dipole moments, results in the isotropic transfer rate, indicated by $\Gamma$ enclosed in angular brackets,

$$
<\Gamma>=\frac{\rho_{f}}{36 \pi \varepsilon_{0}^{2} \hbar R^{6}}\left|\vec{\mu}^{0 m}(\mathrm{D})\right|^{2}\left|\vec{\mu}^{m 0}(\mathrm{~A})\right|^{2}\left[k_{m 0}^{4} R^{4}+k_{m 0}^{2} R^{2}+3\right] .
$$

Like Equation (17), result (18) holds for all pair separation distances $R$ outside the region of overlap of electronic charge distributions associated with $\mathrm{D}$ and $\mathrm{A}$. It therefore excludes stronger contributions 
to the interaction originating from repulsive forces and exchange type of interactions, which dominate at very short D-A displacements. Nonetheless, it is justifiable to claim $[44,46]$, within the context of non-relativistic charged particles interacting with electromagnetic radiation, that molecular QED furnishes a unified theoretical description to be given for the RET process, with the two common mechanisms proposed to understand the transfer process inherent within the results (17) and (18). This becomes readily apparent on extracting the asymptotically limiting forms of the rate. Persisting with the orientationally averaged formula Equation (18), the so-called near- and far-zone rates are obtained as follows.

At short separation distances $k_{m 0} R<<1$, so that the dominant term within square brackets of Equation (18) is the constant term, yielding the near-zone asymptote

$$
<\Gamma>_{\mathrm{NZ}}=\frac{\rho_{f}}{12 \pi \varepsilon_{0}^{2} \hbar R^{6}}\left|\vec{\mu}^{0 m}(\mathrm{D})\right|^{2}\left|\vec{\mu}^{m 0}(\mathrm{~A})\right|^{2},
$$

exhibiting an inverse sixth power dependence on $R$. This was the result obtained by Förster. It is interpreted as arising from radiationless transfer of energy. Inspection of the retarded dipole-dipole coupling tensor Equation (14) shows that the short-range limit Equation (19) may be obtained directly by invoking the near-zone approximation and retaining the contribution independent of $k_{m 0} R$ in $V_{i j}\left(k_{m 0}, \vec{R}\right)$,

$$
V_{i j}^{\mathrm{NZ}}(0, \vec{R})=\frac{1}{4 \pi \varepsilon_{0} R^{3}}\left(\delta_{i j}-3 \hat{R}_{i} \hat{R}_{j}\right),
$$

which amounts to taking the zero-frequency limit, with $\mathrm{e}^{i \omega r / c} \rightarrow 1$ as $\omega \rightarrow 0$, and coincides with $V_{i j}^{\text {static }}$ given in Equation (1), producing for the matrix element in the near-zone,

$$
M_{f i}^{\mathrm{NZ}}=\frac{1}{4 \pi \varepsilon_{0} R^{3}} \mu_{i}^{0 m}(\mathrm{D}) \mu_{j}^{m 0}(\mathrm{~A})\left(\delta_{i j}-3 \hat{R}_{i} \hat{R}_{j}\right),
$$

which is just the static dipolar coupling. Diagrammatically, RET in the near-zone may be pictured as in Figure 2. Due to the close proximity of donor and acceptor entities, propagation of the virtual photon and associated conveyance of energy, is effectively instantaneous. This aspect is denoted in the diagram by the dashed horizontal line.

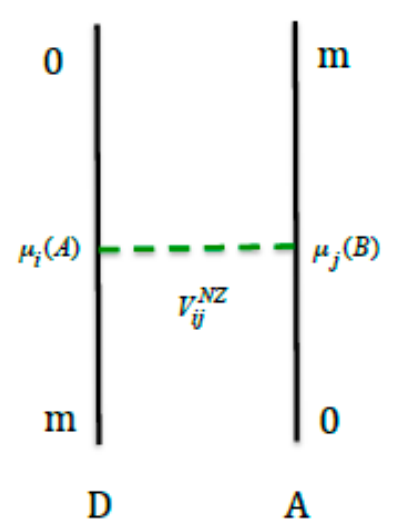

Figure 2. RET in the near-zone.

At the opposite extreme, when $k_{m 0} R>>1$, the dominant contribution to the rate comes from the first term enclosed within square brackets of Equation (18), producing the far-zone limiting form

$$
<\Gamma>_{\mathrm{FZ}}=\frac{\rho_{f} k_{m 0}^{4}}{36 \pi \varepsilon_{0}^{2} \hbar R^{2}}\left|\vec{\mu}^{m 0}(\mathrm{D})\right|^{2}\left|\vec{\mu}^{m 0}(\mathrm{~A})\right|^{2},
$$


which is seen to display an inverse square dependence on separation distance. This is indicative of a radiative transfer mechanism. Even though the photon propagating between centers is virtual, at extremely large separations it possesses all of the characteristics associated with a real photon. This is confirmed by viewing migration of energy as occurring as a result of two uncorrelated events: emission of a photon by the donor particle followed by its absorption some time later by the acceptor species. That the far-zone asymptote of the rate may be obtained and understood in terms of the transmitter-receiver model has been verified by computing, within the framework of molecular QED theory, the intensity of light incident upon the absorption cross-sectional area of A emanating from the irradiance due to spontaneous emission by excited D [44]. Further support for the unmodulated inverse square law limiting expression is provided by calculating the energy flow through a spherical surface of radius $r$ via the Poynting vector [62]. Only the $r^{-1}$ dependent terms of the electric displacement and magnetic fields of an electric dipole source contribute to the energy flux. At displacements beyond wave function overlap, the full rate expression Equation (18) should be employed, containing $R^{-2}$, $R^{-4}$ and $R^{-6}$ dependent terms, with the $R^{-4}$ contribution dominating at intermediate separations.

The RET matrix element and rate have been obtained using different physical viewpoints for picturing the transfer process and computed via alternate calculational schemes. They have confirmed the result using perturbation theory. One of these approaches involves calculating the interaction energy due to the coupling of the electric dipole of $A$ to the electric displacement field of $D$ at the position of A. The causal electric dipole-dependent transverse electric displacement field arising from the source is $[62,65,66]$

$$
d_{i}^{\perp}(\vec{\mu} ; \vec{r}, t)=\frac{1}{4 \pi} \sum_{m, n}\left(-\nabla^{2} \delta_{i j}+\nabla_{i} \nabla_{j}\right) \mu_{j}^{m n}(t-r / c) \frac{e^{i \omega_{m n}(t-r / c)}}{r} .
$$

On making the perturbative approximation by evaluating the electric dipole at $t=0$, the $|0\rangle \leftarrow|m\rangle$ matrix element of the field Equation (23) due to species D, is

$$
\left\langle 0\left|d_{i}^{\perp}(\vec{\mu} ; \vec{r}, t)\right| m\right\rangle=\frac{1}{4 \pi} \mu_{j}^{0 m}(\mathrm{D})\left[\left(\delta_{i j}-3 \hat{r}_{i} \hat{r}_{j}\right)\left(\frac{1}{r^{3}}+\frac{i k_{m 0}}{r^{2}}\right)-\left(\delta_{i j}-\hat{r}_{i} \hat{r}_{j}\right) \frac{k_{m 0}^{2}}{r^{2}}\right] \mathrm{e}^{-i k_{m 0}(r-c t)} .
$$

The coupling of the transition dipole moment of $\mathrm{A}, \mu_{i}^{m 0}(\mathrm{~A})$, to the source driving field of $\mathrm{D}$, evaluated at the position of A relative to $\mathrm{D}, \vec{r}=\vec{R}$, results in the matrix element for RET [24]

$$
\begin{aligned}
& -\varepsilon_{0}^{-1} \mu_{i}^{m 0}(\mathrm{~A}) \mathrm{e}^{-i \omega_{m 0} t}\left\langle 0\left|d_{i}^{\perp}(\vec{\mu} ; \vec{r}, t)\right| m\right\rangle \\
& =\frac{1}{4 \pi \varepsilon_{0} R^{3}} \mu_{j}^{0 m}(\mathrm{D}) \mu_{i}^{m 0}(\mathrm{~A})\left[\left(\delta_{i j}-3 \hat{R}_{i} \hat{R}_{j}\right)\left(1+i k_{m 0} R\right)-\left(\delta_{i j}-\hat{R}_{i} \hat{R}_{j}\right) k_{m 0}^{2} R^{2}\right] \mathrm{e}^{-i k_{m 0} R},
\end{aligned}
$$

which is in agreement with Equation (15).

Historically, as well as for contemporary relevance, it is interesting to note that Förster's original formulation of the transfer rate (valid in the near-zone) was written as a spectral overlap integral,

$$
\Gamma=\frac{9}{8 \pi^{2} c \tau_{D}} \int_{0}^{\infty} I_{\mathrm{D}}(\omega) \sigma_{\mathrm{A}}(\omega)\left|V_{i j}(\omega, \vec{R})\right|^{2} \omega^{2} d \omega,
$$

capturing the essential photophysics of the emitter-absorber model, where $\omega=c k$ is the circular frequency, $I_{\mathrm{D}}(\omega)$ is the emission spectrum of $\mathrm{D}$ and $\sigma_{\mathrm{A}}(\omega)$ is the absorption cross-section of the acceptor atom or molecule, A. Also appearing in Equation (26) is $\tau_{\mathrm{D}}$, the radiative lifetime of the emitter.

\section{Effects of a Medium}

To realistically model energy transfer, the effects of a medium must be included since migration of energy between donor and acceptor rarely takes place in isolation [39,67]. An approach that has 
been developed and is applicable in the condensed phase is to quantize the electromagnetic field together with the particles that comprise the medium but exclude the two particles that are exchanging energy. The photon, the quantized particle of light associated with the free electromagnetic field, is now interpreted as being "dressed" by the surrounding bath and is instead called a polariton [68]. When applied to RET, screening and local field effects are accounted for, with the medium described in terms of a complex refractive index, thereby accommodating dissipative effects there. Treating each bath species as having multiple energy levels with a sufficiently high density so as to form a near-continuum of states results in the polariton possessing a finite lifetime and has the consequence that the transfer rate displays the correct exponential decay term. These aspects manifest themselves in the modification of the vacuum form of the retarded dipole-dipole interaction tensor to a function that is appropriate to excitation exchange in a dielectric medium. Thus

$$
V_{i j}^{\text {med }}(k, \vec{r})=\frac{1}{n^{2}}\left(\frac{n^{2}+2}{3}\right)^{2} V_{i j}(n k, \vec{r}),
$$

where $n=n^{\prime}+i n^{\prime \prime}$ is the complex refractive index of the medium, with imaginary component $n^{\prime \prime}$ describing loss of energy to the environment. $n$ is related to the relative permittivity of the medium $\varepsilon_{r}=\varepsilon / \varepsilon_{0}$ by $n^{2}=\varepsilon_{r}$, with $\varepsilon_{r}$ expressed in terms of the polarizability of the medium, $\alpha$, through the Clausius-Mossotti formula

$$
\varepsilon_{r}=1+\frac{\alpha \rho / \varepsilon_{0}}{1-\alpha \rho / 3 \varepsilon_{0}}
$$

with $\rho$ the density of the medium. The local field factor and dielectric screening effect change the influence of the vacuum form of the retarded coupling tensor in a medium through the pre-factors in Equation (27). Furthermore, it is interesting to note that the wave number is now scaled by the refractive index, that is, $k \rightarrow n k$. Unsurprisingly, the rate Equation (18) is modified significantly due to the effects of the environment. There are now terms explicitly dependent upon the real and imaginary parts of the refractive index; this in turn gives rise to inverse cubic and inverse fifth power dependent contributions on $R$, as well as modified $R^{-2}, R^{-4}$ and $R^{-6}$ dependent terms. Explicit formulae are given by Equation (4.38) of Ref. [69] and Equation (17) of Ref. [70].

The analogue of Equation (26) in a medium is then

$$
\Gamma^{\text {med }}=\frac{9}{8 \pi c^{2} \tau_{D}} \int_{0}^{\infty} I_{\mathrm{D}}^{\operatorname{med}}(\omega) \sigma_{\mathrm{A}}^{\operatorname{med}}(\omega)\left|V_{i j}^{\operatorname{med}}(\omega, \vec{R})\right|^{2} \omega^{2} \mathrm{e}^{-2 n^{\prime \prime} \omega R / c} d \omega,
$$

where the modified forms of the emission spectrum of $\mathrm{D}$ and absorption cross-section of $\mathrm{A}$ are given by

$$
I_{\mathrm{D}}^{\operatorname{med}}(\omega)=n^{\prime}\left|\frac{n^{2}+2}{3}\right|^{2} I_{\mathrm{D}}(\omega)
$$

and

$$
\sigma_{\mathrm{A}}^{\operatorname{med}}(\omega)=\frac{1}{n^{\prime}}\left|\frac{n^{2}+2}{3}\right|^{2} \sigma_{\mathrm{A}}(\omega) .
$$

Note the explicit presence of the dissipative factor in Equation (29). In the near-zone, $|n| k R<<1$ and the Förster type $R^{-6}$ term again dominates, while radiative $R^{-2}$ behavior is found in the far-zone, when $|n| k R>>1$; the decomposition into distinct emission and absorption events is nicely illustrated by expressions (30). Known results for migration of energy in a vacuum are recovered on inserting $n=1$.

\section{Effect of Higher Multipoles}

Up to now, transfer of energy has only been considered between an electric dipole donor and an acceptor species that is also able to undergo an electric dipole allowed transition. This is 
reflected in Equation (7), the coupling Hamiltonian, which was restricted to terms satisfying the long wavelength approximation, thereby permitting electric dipole interaction terms only. For many species, however, this assumption is too restrictive. Spatial variations of the vector potential then have to be accounted for as the entity interacting with electromagnetic radiation no longer experiences uniform electric and magnetic fields. This results in higher-order multipole contributions being added to the leading electric dipole coupling term. These are not only limited to electric quadrupole and octupole moments but can also include magnetic dipole and diamagnetic terms. Coupling between matter and radiation in the multipolar version of molecular QED theory automatically includes the interaction of electric polarization and magnetization distributions with electric displacement and magnetic fields, respectively. Adding the next few terms of the multipole expansion extends Equation (7) for a single molecule, $\xi$, to

$$
\begin{aligned}
& H_{\text {int }}(\xi)=-\varepsilon_{0}^{-1} \mu_{i}(\xi) d_{i}^{\perp}\left(\vec{R}_{\xi}\right)-\varepsilon_{0}^{-1} Q_{i j}(\xi) \nabla_{j} d_{i}^{\perp}\left(\vec{R}_{\xi}\right)-\varepsilon_{0}^{-1} O_{i j k}(\xi) \nabla_{j} \nabla_{k} d_{i}^{\perp}\left(\vec{R}_{\xi}\right)-\ldots \\
& -\vec{m}(\xi) \cdot \vec{b}\left(\vec{R}_{\xi}\right)+\ldots+\frac{e^{2}}{8 m}\left\{\vec{q} \times \vec{b}\left(\vec{R}_{\xi}\right)\right\}^{2}+\ldots
\end{aligned}
$$

In Equation (31), $Q_{i j}(\xi)$ is the electric quadrupole moment operator, which couples to the gradient of the electric displacement field and $O_{i j k}(\xi)$ is the electric octupole moment operator. The leading order magnetic interaction is described by the magnetic dipole moment operator, $\vec{m}(\xi)$, which interacts linearly with the magnetic field, $\vec{b}(\vec{r})$, and the last term written explicitly in Equation (31), which is the diamagnetic coupling and which is proportional to the square of $\vec{b}(\vec{r})$, with $\vec{q}$ the generalized coordinate variable. Analogous to Equation $(8), \vec{b}(\vec{r})$ may be expressed as a Fourier series expansion in the field modes:

$$
\vec{b}(\vec{r})=i \sum_{\vec{k}, \lambda}\left(\frac{\hbar k}{2 \varepsilon_{0} c V}\right)^{1 / 2}\left[\vec{b}^{(\lambda)}(\vec{k}) a^{(\lambda)}(\vec{k}) \mathrm{e}^{i \vec{k} \cdot \vec{r}}-\overline{\vec{b}}^{(\lambda)}(\vec{k}) a^{\dagger(\lambda)}(\vec{k}) \mathrm{e}^{-i \vec{k} \cdot \vec{r}}\right]
$$

where $\vec{b}^{(\lambda)}(\vec{k})=\hat{k} \times \vec{e}^{(\lambda)}(\vec{k})$ is the complex unit magnetic polarization vector, with the triad of vectors $\vec{e}^{(\lambda)}(\vec{k}), \vec{b}^{(\lambda)}(\vec{k})$, and $\hat{\vec{k}}$ forming a right-handed set.

Instances when the electric dipole approximation has to be relaxed include situations where the size of the chromophore is large enough that it sees a spatially non-uniform electromagnetic field. Also, species possessing no symmetry and belonging to the $C_{1}$ point group permit transitions to all multipole orders. Similarly, molecules lacking an improper axis of rotation, which are termed chiral, have few or no elements of symmetry and consequently have spectroscopic selection rules that are less restrictive. To leading order such species simultaneously allow electric dipole and quadrupole and magnetic dipole transitions to occur.

Here we consider resonant exchange of energy between two optically active or chiral molecules and retain electric and magnetic dipole coupling terms in the interaction Hamiltonian, Equation (31). Hence

$$
H_{\text {int }}(\xi)=-\varepsilon_{0}^{-1} \vec{\mu}(\xi) \cdot \vec{d}^{\perp}\left(\vec{R}_{\xi}\right)-\vec{m}(\xi) \cdot \vec{b}\left(\vec{R}_{\xi}\right),
$$

with $H_{\text {int }}=\sum_{\xi=\mathrm{D}, \mathrm{A}} H_{\text {int }}(\xi)$. The initial and final states for the problem are the same as in the electric dipole case studied earlier and given by Equation (9). A single virtual photon is again exchanged between the two units, so that the expression for the second-order matrix element, Equation (10), may be re-employed. Since the interaction vertex may be of either the electric dipole type or of the magnetic dipole variety, the number of possible time-ordered sequences of single virtual photon exchange between the two chiral species doubles from two to four compared to pure electric dipole transfer. 
In order to evaluate the transfer rate, the contribution to the matrix element arising from exchange of a virtual photon between an electric dipole at one center and a magnetic dipole at the second and vice versa, is required [71]. This is given by

$$
M_{f i}^{\mu m}+M_{f i}^{m \mu}=\left\{\mu_{i}^{0 m}(\mathrm{D}) m_{j}^{m 0}(\mathrm{~A})+m_{j}^{0 m}(\mathrm{D}) \mu_{i}^{m 0}(\mathrm{~A})\right\} U_{i j}\left(k_{m 0}, \vec{R}\right),
$$

where the retarded resonant tensor coupling electric and magnetic dipoles is given by

$$
U_{i j}(k, \vec{r})=-\frac{i k}{4 \pi \varepsilon_{0} c} \varepsilon_{i j k} \nabla_{k} \frac{e^{i k r}}{r}=\frac{1}{4 \pi \varepsilon_{0} c r^{3}} \varepsilon_{i j k} \hat{r}_{k}\left(i k r+k^{2} r^{2}\right) \mathrm{e}^{i k r},
$$

and $\varepsilon_{i j k}$ is the Levi-Civita tensor. It is seen that $U_{i j}(k, \vec{r})$ is purely retarded.

The total matrix element is a sum of contributions from electric dipole-electric dipole, magnetic dipole-magnetic dipole, electric dipole-magnetic dipole and magnetic dipole-electric dipole terms, so that the total Fermi golden rule rate is

$$
\Gamma=\frac{2 \pi \rho_{f}}{\hbar}\left|M_{f i}^{\mu \mu}+M_{f i}^{m m}+M_{f i}^{\mu m}+M_{f i}^{m \mu}\right|^{2}
$$

A discriminatory transfer rate originates from two sources: one from the interference of pure electric and pure magnetic terms, $M_{f i}^{\mu \mu}$ and $M_{f i}^{m m}$, while the second arises from the modulus square of the mixed electric-magnetic contribution [71,72]. Their addition results in the Fermi rate

$$
<\Gamma^{\text {chiral }}>=-\frac{\rho_{f}}{18 \pi \varepsilon_{0}^{2} \hbar c^{2} R^{6}}\left[\vec{\mu}^{0 m}(\mathrm{D}) \cdot \vec{m}^{m 0}(\mathrm{D})\right]\left[\vec{\mu}^{0 m}(\mathrm{~A}) \cdot \vec{m}^{m 0}(\mathrm{~A})\right]\left[3+2 k_{m 0}^{2} R^{2}+2 k_{m 0}^{4} R^{4}\right]
$$

applicable for randomly oriented chiral molecules and for separations beyond orbital overlap.

An especially interesting feature of the result Equation (37) is its discriminatory behavior, depending on the handedness of each species. This manifests in the rate formula through its dependence on the pseudoscalar quantity $\vec{\mu}^{0 m}(\xi) \cdot \vec{m}^{m 0}(\xi)$, which takes an opposite sign for a left-handed enantiomer versus its right-handed form. Thus, replacing one chiral molecule by its antipodal form changes the sign of the transfer rate.

Asymptotic limits follow from result (37) in the usual way. In the near-zone, when $k_{m 0} R<<1$, the term independent of $k_{m 0} R$ in the polynomial portion is dominant, leading to

$$
<\Gamma^{\text {chiral }}>_{\mathrm{NZ}}=-\frac{\rho_{f}}{6 \pi \varepsilon_{0}^{2} \hbar c^{2} R^{6}}\left[\vec{\mu}^{0 m}(\mathrm{D}) \cdot \vec{m}^{m 0}(\mathrm{D})\right]\left[\vec{\mu}^{0 m}(\mathrm{~A}) \cdot \vec{m}^{m 0}(\mathrm{~A})\right],
$$

and which exhibits a Förster like inverse sixth power dependence. At large donor-acceptor displacements, for which $k_{m 0} R>>1$, an expected inverse square radiative rate formula ensues,

$$
<\Gamma^{\mathrm{chiral}}>_{\mathrm{FZ}}=-\frac{\rho_{f} k_{m 0}^{4}}{9 \pi \varepsilon_{0}^{2} \hbar c^{2} R^{2}}\left[\vec{\mu}^{0 m}(\mathrm{D}) \cdot \vec{m}^{m 0}(\mathrm{D})\right]\left[\vec{\mu}^{0 m}(\mathrm{~A}) \cdot \vec{m}^{m 0}(\mathrm{~A})\right]
$$

indicative of real photon emission followed by absorption. Contributions to the discriminatory rate of a similar order of magnitude arising from electric quadrupole and octupole coupling have also been calculated $[73,74]$.

\section{The Role of an Additional Body}

Thus far the presentation has been limited to migration of energy between two centers. The donor and acceptor could be the only two particles situated in a vacuum, or surrounded by a large number of microscopic objects that form a medium with uniform dielectric constant. The latter was considered 
earlier in terms of the polariton exchange model, with explicit account being taken of the complex refractive index of the surrounding bath.

From the microscopic perspective, the theory of RET may be extended by exploring the influence of an additional atom or molecule in modifying the transfer rate. A common assumption that is made is to take the third particle to be a neutral, polarizable species that relays energy between donor and acceptor and playing no other role. Its influence is explicitly accounted for by including its presence in the total Hamiltonian for the system. Equation (3) now becomes

$$
H=\sum_{\xi=\mathrm{D}, \mathrm{A}, \mathrm{C}} H_{\mathrm{mol}}(\xi)+H_{\mathrm{rad}}+\sum_{\xi=\mathrm{D}, \mathrm{A}, \mathrm{C}} H_{\mathrm{int}}(\xi)
$$

where $C$ denotes the third body, which is positioned at $\vec{R}_{\mathrm{C}}$. Staying within the electric dipole approximation, Equation (7) has an additional term representing the coupling of $C$ through its electric dipole moment, $\vec{\mu}(\mathrm{C})$, to the transverse electric displacement field, $\vec{d}^{\perp}\left(\vec{R}_{\mathrm{C}}\right)$. The two-particle initial and final states Equation (9) are trivially modified to account for $C$, as in

$$
|i\rangle=\left|m^{\mathrm{D}}, 0^{\mathrm{A}}, 0^{\mathrm{C}}\right\rangle,
$$

and

$$
|f\rangle=\left|0^{\mathrm{D}}, m^{\mathrm{A}}, 0^{\mathrm{C}}\right\rangle \text {. }
$$

With $\mathrm{C}$ serving as a bridge between $\mathrm{D}$ and $\mathrm{A}$, it is coupled to both particles via the exchange of a single virtual photon, that between D and C of mode $\vec{p}, \varepsilon$ and that traversing between $C$ and $A$ labelled $\vec{p}^{\prime}, \varepsilon^{\prime}$. In this problem twenty-four time-ordered diagrams have to be evaluated and summed over at fourth order of perturbation theory. A representative diagram is shown in Figure 3, in which a $\vec{p}, \varepsilon$ virtual photon propagates from $\mathrm{C}$ to $\mathrm{D}$, followed by the $\vec{p}^{\prime}, \varepsilon^{\prime}$ virtual photon being emitted by $\mathrm{C}$ and then absorbed by A. Species $\mathrm{C}$ undergoes electronic excitation to virtual levels $|q\rangle$. The third particle is positioned at the origin, with $\mathrm{D}$ and A displaced with respect to $C$ by $\vec{\rho}$ and $\vec{\rho}^{\prime}$, that is $\vec{\rho}=\vec{R}_{\mathrm{D}}-\vec{R}_{\mathrm{C}}$ and $\vec{\rho}^{\prime}=\vec{R}_{\mathrm{A}}-\vec{R}_{\mathrm{C}}$, so that $\vec{\rho}^{\prime}-\vec{\rho}=\vec{R}_{\mathrm{A}}-\vec{R}_{\mathrm{D}}=\vec{R}$. The matrix element is $[5,62,75,76]$

$$
M_{f i}^{\mathrm{DCA}}=-\mu_{i}^{0 m}(\mathrm{D}) \alpha_{k l}^{\mathrm{C}}(-k, k) \mu_{j}^{m 0}(\mathrm{~A}) V_{i k}(k, \vec{\rho}) V_{j l}\left(k, \vec{\rho}^{\prime}\right),
$$

where $k=k_{m 0}$. The effect of the third species manifests through its dynamic polarizability tensor at the wave number of the resonant transition, the opposite signs of $k$ in the argument illustrating the scattered nature of the two virtual photons [55]. The general form of the polarizability of molecule $\xi$ is defined for an $|s\rangle \leftarrow|r\rangle$ transition as

$$
\alpha_{i j}^{r s}\left(\xi ; \mp k_{1}, \mp k_{2}\right)=\sum_{q}\left\{\frac{\mu_{i}^{s q}(\xi) \mu_{j}^{q r}(\xi)}{E_{q r} \pm \hbar c k_{1}}+\frac{\mu_{j}^{s q}(\xi) \mu_{i}^{q r}(\xi)}{E_{q r} \pm \hbar c k_{2}}\right\} .
$$

Exchange of a virtual photon between D and C is represented by $V_{i k}(k, \vec{\rho})$ in Equation (42), while the second coupling tensor accounts for relay of excitation energy between $C$ and $A$.

The total matrix element is therefore a sum of the direct D-A contribution Equation (15) and that due to the presence of C, Equation (42), resulting in three distinct contributions to the transfer rate,

$$
\Gamma=\frac{2 \pi \rho_{f}}{\hbar}\left|M_{f i}^{\mathrm{DA}}+M_{f i}^{\mathrm{DCA}}\right|^{2}=\Gamma^{\mathrm{d}}+\Gamma^{\mathrm{i}}+\Gamma^{\mathrm{int}} .
$$




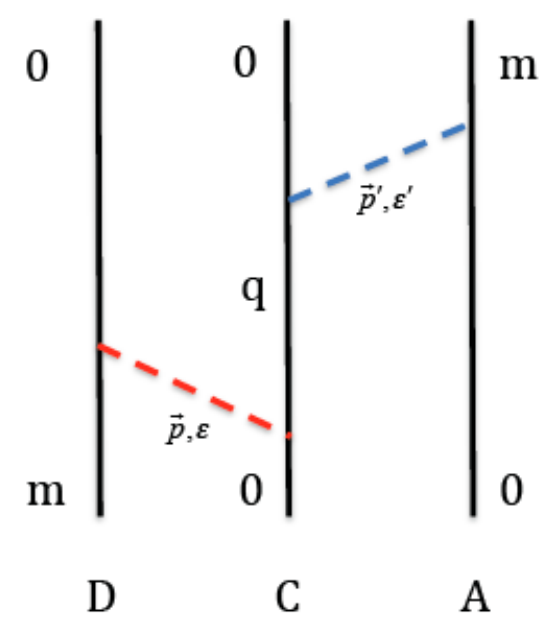

Figure 3. One of 24 possible time-orderings for RET mediated by a third particle, $C$, acting as a bridge between $D$ and $A$.

$\Gamma^{\mathrm{d}}$ is the direct contribution to the total rate, given by Equations (17) and (18) for oriented and isotropic systems, respectively. The indirect contribution to the rate, $\Gamma^{\mathrm{i}}$, that involving $\mathrm{C}$, is proportional to $\left|M_{f i}^{\mathrm{DCA}}\right|^{2}$, while the interference term between direct and indirect pathways is represented by

$$
\Gamma^{\mathrm{int}}=\frac{4 \pi \rho_{f}}{\hbar} \operatorname{Re} \bar{M}_{f i}^{\mathrm{DA}} M_{f i}^{\mathrm{DCA}} .
$$

Detailed forms for the last two summands in Equation (44) valid for all $\rho$ and $\rho^{\prime}$ may be found in Ref. [76], extending earlier work by Craig and Thirunamachandran, Ref. [62], that was limited to short D-C and C-A separations.

For species in the fluid phase and that are close to one another, so that the near-zone limit holds, $<\Gamma^{\mathrm{i}}>_{\mathrm{NZ}}$ exhibits an inverse sixth power dependence on each of $\rho$ and $\rho^{\prime}$, while $<\Gamma^{\text {int }}>_{\mathrm{NZ}}$ is proportional to $\left(\rho \rho^{\prime} R\right)^{-3}$. For C positioned mid-way between $\mathrm{D}$ and $\mathrm{A}$, with all three bodies lying on a straight line, the rate displays an inverse ninth power separation distance dependence that, interestingly, is negative in sign, meaning that the total transfer rate is reduced. Inverse square dependences on $\rho$ and $\rho^{\prime}$ are found to occur for $<\Gamma^{\mathrm{i}}>_{\mathrm{FZ}}$ at very large displacements, corresponding to the far-zone limit. In contrast, the interference contribution varies inversely with respect to $\rho, \rho^{\prime}$ and $R$ in this limit.

Order of magnitude estimates indicate that $\left.\left\langle\Gamma^{\mathrm{i}}\right\rangle_{\mathrm{NZ}} \approx 10<\Gamma^{\mathrm{i}}\right\rangle_{\mathrm{NZ}}$, from which it may be concluded that the effect of a third body in shuttling energy between donor and acceptor is enhanced when all three entities are in close proximity to each other.

The third body need not only serve in the role of a bridging species but may facilitate transfer of energy between donor and acceptor by coupling to only one of the pair and not the other. These two additional third-body mediated mechanisms, which may be designated as the CDA or DAC pathways, have been studied by Daniels and Andrews [75] and by Andrews and Ford [77] and by Ford and Andrews [78]. The Hamiltonian operator and initial and final states for the other two possible mechanisms are identical to that for the DCA scheme, given by Equations (40) and (41). Twenty-four time-ordered sequences of virtual photon emission and absorption may be drawn for each mechanism. In the first case a single virtual photon of mode $\vec{p}, \varepsilon$ is exchanged between $C$ and $D$, while the virtual photon coupling D and A is of mode $\vec{p}^{\prime}, \varepsilon^{\prime}$. In the second scenario D and A are coupled via a $\vec{p}, \varepsilon$ mode photon, with a $\vec{p}^{\prime}, \varepsilon^{\prime}$ mode virtual photon traversing between A and C. Fourth-order perturbation theory again yields the matrix elements, which are [79]

$$
M_{f i}^{\mathrm{CDA}}=\mu_{i}^{00}(\mathrm{C}) \alpha_{k l}^{0 m}(\mathrm{D} ; k, 0) \mu_{j}^{m 0}(\mathrm{~A}) V_{i k}(0, \vec{\rho}) V_{j l}(k, \vec{R}),
$$


and

$$
M_{f i}^{\mathrm{DAC}}=\mu_{j}^{0 m}(\mathrm{D}) \alpha_{k l}^{m 0}(\mathrm{~A} ;-k, 0) \mu_{i}^{00}(\mathrm{C}) V_{i k}\left(0, \vec{\rho}^{\prime}\right) V_{j l}(k, \vec{R}) .
$$

Note that the polarizabilities appearing in expressions (46) and (47) are transition polarizabilities between ground and excited state $\mid m>$. Evident from these two matrix elements is that $C$ couples with the donor in the first case and with the acceptor in the second case, through its ground state permanent electric dipole moment, $\vec{\mu}^{00}$ (C). Therefore the third body, C, must be polar. A direct consequence is that no real energy is transferred between $C$ and $D$ in the CDA route, or between $C$ and $A$ in the DAC pathway. This results in the appearance of the static dipolar coupling potentials in Equations (46) and (47). Furthermore, the polarizability of the donor shows up in the CDA mechanism, while that of the acceptor features in Formula (47), with one frequency component vanishing in each case. Expressions for the rate arising from the two additional coupling schemes, as well as interference terms involving the pair mechanism and individual three-body exchange pathways, have been obtained and examined in the near-zone [77].

If selection rules forbid a one-photon allowed transition in D, both the DCA and DAC mechanisms are inoperative, leaving Equation (46) as the sole three-body mediated contribution to the rate. Only Equation (47) survives, however, if a one-photon allowed transition is forbidden in the acceptor moiety. Whether transfer rates obtained directly from Equations (46) and (47) are non-vanishing depend on two-photon selection rules for the donor and acceptor, respectively, as they determine if the respective polarizability is non-zero or not. When the transition dipoles of D and A and their separation distance are mutually perpendicular, only the relay mechanism DCA contributes to the three-body transfer rate. Other configurations allow appreciable enhancements or reductions in the total rate correct up to three-body terms [75].

The molecular QED theory of direct (two-body) and indirect (third-body mediated) RET summarized above has been applied to nanostructures with differing dimensionalities, including quantum wells, nanowires [5] and quantum dots [3,6].

\section{Summary and Outlook}

Application of the theory of molecular QED to RET has been reviewed. Energy is relayed from donor to acceptor species via the exchange of a single virtual photon. Such quanta of radiation are permitted by QED and in general mediate the interaction between material particles. Second-order perturbation theory yields the matrix element for resonant transfer between identical atoms or molecules, from which the probability and Fermi golden rule transfer rate readily follow. The rate holds for all pair separation distances outside the orbital overlap region and is proportional to the squares of the transition electric dipole moments of each particle. Radiationless transfer of energy, having inverse sixth power dependence on displacement and associated with Förster follows as the near-zone limiting form of the general rate formula and corresponds to instantaneous signal propagation between the two sites. At large separations, on the other hand, the rate displays characteristic inverse square dependence, indicative of a radiative transfer mechanism in which the donor decays by spontaneous emission of a photon, which is then absorbed at the space-time point of the acceptor species, the two photonic events being uncorrelated, with the virtual photon becoming a real one in the limit $R \rightarrow \infty$.

To account for the effect of a reservoir of bath molecules on the transfer rate, the polariton was invoked. This is a medium dressed photon and the retarded coupling tensor is modified from its vacuum form through the appearance of a pre-factor that describes local field effects and screening contributions through the complex refractive index of the medium, which correspondingly scales the free-field frequency. In addition to $R^{-2}, R^{-4}$ and $R^{-6}$ dependent terms as found in transfer taking place in a vacuum, the rate has $R^{-3}$ and $R^{-5}$ terms. Discriminatory transfer rates, arising when energy is exchanged between optically active enantiomers, was also described and could be treated as a straightforward extension of electric dipole-electric dipole transfer by including magnetic dipole 
coupling terms in the interaction Hamiltonian and extracting terms in the rate proportional to the pseudoscalar quantity $\vec{\mu}^{0 t}(\xi) \cdot \vec{m}^{t 0}(\xi)$ of the chiral molecule $\xi$.

With pair transfer now very well understood, in recent years effort has been targeted to examining the effect of a third, polarizable molecule that mediates exchange between donor and acceptor but which remains unchanged overall. Computational complexity increases significantly on addition of one extra species, even if its role is entirely passive. Contributing to this difficulty is the fact that two pathways involving the third body are now possible in addition to the one that is expected to be the most important, that in which the third particle in close proximity serves as a bridge between D and A.

Future work involves studying the effect of two additional passive species on the total RET rate, again at short separations, which together with the result covering three particles, will enable insight to be gained into microscopic versus macroscopic approaches to RET modified by a medium. Furthermore, higher-order corrections to RET occurring between donor and acceptor through the exchange of more than virtual photon could also be studied. As well as advances in basic theory, applications are expected to continue, with particular focus in the areas of biophysics [80] and nanoscience [8].

Funding: This research received no external funding.

Conflicts of Interest: The author declares no conflict of interest.

\section{References}

1. May, V.; Kühn, O. Charge and Energy Transfer Dynamics in Molecular Systems; Wiley-VCH: Weinheim, Germany, 2011.

2. $\quad$ Andrews, D.L.; Demidov, A.A. (Eds.) Resonance Energy Transfer; Wiley: Chichester, UK, 1999.

3. Scholes, G.D.; Andrews, D.L. Resonance Energy Transfer and Quantum Dots. Phys. Rev. B 2005, 72, 125331. [CrossRef]

4. Olaya-Castro, A.; Scholes, G.D. Energy Transfer from Förster-Dexter Theory to Quantum Coherent Light-Harvesting. Int. Rev. Phys. Chem. 2011, 30, 49-77. [CrossRef]

5. Weeraddana, D.; Premaratne, M.; Andrews, D.L. Direct and Third-Body Mediated Resonance Energy Transfer in Dimensionally Constrained Nanostructures. Phys. Rev. B 2015, 92, 035128. [CrossRef]

6. Weeraddana, D.; Premaratne, M.; Andrews, D.L. Quantum Electrodynamics of Resonance Energy Transfer in Nanowire Systems. Phys. Rev. B 2016, 93, 075151. [CrossRef]

7. Rolczynski, B.S.; Navotnaya, P.; Sussman, H.R.; Engel, G.S. Cysteine-Mediated Mechanism Disrupts Energy Transfer to Prevent Photooxidation. Proc. Natl. Acad. Sci. USA 2016, 113, 8562-8564. [CrossRef] [PubMed]

8. Hsu, L.-Y.; Ding, W.; Schatz, G.C. Plasmon-Coupled Resonance Energy Transfer. J. Phys. Chem. Lett. 2017, 8, 2357-2367. [CrossRef] [PubMed]

9. Zhong, X.; Chervy, T.; Zhang, L.; Thomas, A.; George, J.; Genet, C.; Hutchison, J.; Ebbesen, T.W. Energy Transfer between Spatially Separated Entangled Molecules. Angew. Chem. Int. Ed. 2017, 56, 9034-9038. [CrossRef] [PubMed]

10. Heitler, W.; London, F. Wechselwirkung neutraler Atome und homöopolare Bindung nach der Quantenmechanik. Z. Phys. 1927, 44, 455-472. [CrossRef]

11. Eisenschitz, R.; London, F. Über das Verhältnis der van der Waalsschen Kräfte zu den homöopolaren Bindungskräften. Z. Phys. 1930, 60, 491-527. [CrossRef]

12. Heisenberg, W.; Pauli, W. Zur Quantendynamik der Wellenfelder. Z. Phys. 1929, 56, 1-61. [CrossRef]

13. Heisenberg, W.; Pauli, W. Zur Quantendynamik der Wellenfelder II. Z. Phys. 1930, 59, 168-190. [CrossRef]

14. Dirac, P.A.M.; Fock, V.A.; Podolsky, B. On Quantum Electrodynamics. Phys. Z. Sowjetunion 1932, 6, 468-479.

15. Fermi, E. Quantum Theory of Radiation. Rev. Mod. Phys. 1932, 4, 87-132. [CrossRef]

16. Kikuchi, S. Über die Fortfpflanzung von Lichtwellen in der Heisenberg-Paulischen Formulierung der Quantenelecktrodynamik. Z. Phys. 1930, 66, 558-571. [CrossRef]

17. Förster, T. Zwischenmolekulare Energiewanderung und Fluoreszenz. Ann. Phys. 1948, 437, 55-75. [CrossRef]

18. Heitler, W.; Ma, S.T. Quantum Theory of Radiation Damping for Discrete States. Proc. R. Irish Acad. 1949, A52, 109-125. 
19. Simpson, W.T. Theory of the Interaction of Localized Electronic Excitations. Rad. Res. 1963, 20, 87-100. [CrossRef]

20. McLone, R.R.; Power, E.A. On the Interaction Between Two Identical Neutral Dipole Systems, One in the Excited State and the Other in the Ground State. Mathematika 1964, 11, 91-94. [CrossRef]

21. Avery, J.S. Resonance Energy Transfer and Spontaneous Photon Emission. Proc. Phys. Soc. 1966, 88, 1-8. [CrossRef]

22. Milonni, P.W.; Knight, P.L. Retardation in the Resonant Interaction of Two Identical Atoms. Phys. Rev. A 1974, 10, 1096-1108. [CrossRef]

23. Shirokov, M.I. Signal Velocity in Quantum Electrodynamics. Sov. Phys. Uspekhi 1978, 21, 345-358. [CrossRef]

24. Power, E.A.; Thirunamachandran, T. Quantum Electrodynamics with Non-Relativistic Sources. III. Intermolecular Interactions. Phys. Rev. A 1983, 28, 2671-2675. [CrossRef]

25. Rubin, M.H. Violation of Einstein Causality in a Model Quantum System. Phys. Rev. D 1987, 35, 3836-3839. [CrossRef]

26. Biswas, A.K.; Compagno, G.; Palma, G.M.; Passante, R.; Persico, F. Virtual Photons and Causality in the Dynamics of a Pair of Two-Level Atoms. Phys. Rev. A 1990, 42, 4291-4301. [CrossRef] [PubMed]

27. Valentini, A. Non-Local Correlations in Quantum Electrodynamics. Phys. Lett. A 1991, 153, $321-325$. [CrossRef]

28. Craig, D.P.; Thirunamachandran, T. An Analysis of Models for Resonant Transfer of Excitation Using Quantum Electrodynamics. Chem. Phys. 1992, 167, 229-240. [CrossRef]

29. Hegerfeldt, G.C. Causality Problems for Fermi's Two-Atom System. Phys. Rev. Lett. 1994, 72, $596-599$. [CrossRef] [PubMed]

30. Milonni, P.W.; James, D.F.V.; Fearn, H. Photodetection and Causality in Quantum Optics. Phys. Rev. A 1995, 52, 1525-1537. [CrossRef] [PubMed]

31. Berman, P.R.; Dubetsky, B. Causality in the Excitation Exchange Between Identical Atoms. Phys. Rev. A 1997, 55, 4060-4069. [CrossRef]

32. Power, E.A.; Thirunamachandran, T. Analysis of the Causal Behaviour in Energy Transfer Between Atoms. Phys. Rev. A 1997, 56, 3395-3408. [CrossRef]

33. Berman, P.R. Interaction Energy of Non-Identical Atoms. Phys. Rev. A 2015, 91, 042127. [CrossRef]

34. Milonni, P.W.; Rafsanjani, S.M.H. Distance Dependence of Two-Atom Dipole Interactions with One Atom in an Excited State. Phys. Rev. A 2015, 92, 062711. [CrossRef]

35. Safari, H.; Karimpour, M.R. Body-Assisted van der Waals Interaction Between Excited Atoms. Phys. Rev. Lett. 2015, 114, 013201. [CrossRef] [PubMed]

36. Donaire, M. Two-Atom Interaction Energies with One Atom in an Excited State: Van der Waals Potentials Versus Level Shifts. Phys. Rev. A 2016, 93, 052706. [CrossRef]

37. Barcellona, P.; Passante, R.; Rizzuto, L.; Buhmann, S.Y. van der Waals Interactions Between Excited Atoms in Generic Environments. Phys. Rev. A 2016, 94, 012705. [CrossRef]

38. Dexter, D.L. A Theory of Sensitized Luminescence in Solids. J. Chem. Phys. 1953, 21, 836-850. [CrossRef]

39. Juzeliunas, G.; Andrews, D.L. Quantum Electrodynamics of Resonance Energy Transfer. Adv. Chem. Phys. 2000, 112, 357-410.

40. Cohen, A.E.; Mukamel, S. A Mechanical Force Accompanies Fluorescence Resonance Energy Transfer (FRET). J. Phys. Chem. A 2003, 107, 3633-3638. [CrossRef]

41. Scholes, G.D. Long-Range Resonance Energy Transfer in Molecular Systems. Ann. Rev. Phys. Chem. 2003, 54, 57-87. [CrossRef] [PubMed]

42. Schwinger, J.S. (Ed.) Selected Papers on Quantum Electrodynamics; Dover: New York, NY, USA, 1958.

43. Andrews, D.L.; Jones, G.A.; Salam, A.; Woolley, R.G. Perspective: Quantum Hamiltonians for Optical Interactions. J. Chem. Phys. 2018, 148, 040901. [CrossRef] [PubMed]

44. Andrews, D.L. A Unified Theory of Radiative and Radiationless Molecular Energy Transfer. Chem. Phys. 1989, 135, 195-210. [CrossRef]

45. Andrews, D.L. Mechanistic Principles and Applications of Resonance Energy Transfer. Can. J. Chem. 2008, 86, 855-870. [CrossRef]

46. Daniels, G.J.; Jenkins, R.D.; Bradshaw, D.S.; Andrews, D.L. Resonance Energy Transfer: The Unified Theory Revisited. J. Chem. Phys. 2003, 119, 2264-2274. [CrossRef] 
47. Andrews, D.L.; Bradshaw, D.S. The Role of Virtual Photons in Nanoscale Photonics. Ann. Phys. 2014, 526, 173-186. [CrossRef]

48. Grinter, R.A.; Jones, G.A. Resonance Energy Transfer: The Unified Theory via Vector Spherical Harmonics. J. Chem. Phys. 2016, 145, 074107. [CrossRef] [PubMed]

49. Craig, D.P.; Thirunamachandran, T. Molecular Quantum Electrodynamics; Dover: New York, NY, USA, 1998.

50. Salam, A. Molecular Quantum Electrodynamics; John Wiley \& Sons, Inc.: Hoboken, NJ, USA, 2010.

51. Salam, A. Non-Relativistic QED Theory of the van der Waals Dispersion Interaction; Springer: Cham, Switzerland, 2016.

52. Andrews, D.L.; Allcock, P. Optical Harmonics in Molecular Systems; Wiley-VCH: Weinhein, Germany, 2002.

53. Andrews, D.L.; Sherborne, B.S. Resonant Excitation Transfer: A Quantum Electrodynamical Study. J. Chem. Phys. 1987, 86, 4011-4017. [CrossRef]

54. Jenkins, R.D.; Daniels, G.J.; Andrews, D.L. Quantum Pathways for Resonance Energy Transfer. J. Chem. Phys. 2004, 120, 11442-11448. [CrossRef] [PubMed]

55. Salam, A. Virtual Photon Exchange, Intermolecular Interactions and Optical Response Functions. Mol. Phys. 2015, 113, 3645-3653. [CrossRef]

56. Casimir, H.B.G.; Polder, D. The Influence of Retardation on the London van der Waals Forces. Phys. Rev. 1948, 73, 360-372. [CrossRef]

57. Alligood, B.W.; Salam, A. On the Application of State Sequence Diagrams to the Calculation of the Casimir-Polder Potential. Mol. Phys. 2007, 105, 395-404. [CrossRef]

58. Feynman, R.P. The Theory of Positrons. Phys. Rev. 1949, 76, 749-759. [CrossRef]

59. Feynman, R.P. Space-Time Approach to Quantum Electrodynamics. Phys. Rev. 1949, 76, 769-789. [CrossRef]

60. Ward, J.F. Calculation of Nonlinear Optical Susceptibilities Using Diagrammatic Perturbation Theory. Rev. Mod. Phys. 1965, 37, 1-18. [CrossRef]

61. Jenkins, R.D.; Andrews, D.L.; Davila Romero, L.C. A New Diagrammatic Methodology for Non-Relativistic Quantum Electrodynamics. J. Phys. B At. Mol. Opt. Phys. 2002, 35, 445-468. [CrossRef]

62. Craig, D.P.; Thirunamachandran, T. Third-Body Mediation of Resonance Coupling Between Identical Molecules. Chem. Phys. 1989, 135, 37-48. [CrossRef]

63. Frost, J.E.; Jones, G.A. A Quantum Dynamical Comparison of the Electronic Couplings Derived from Quantum Electrodynamics and Förster Theory: Application to 2D Molecular Aggregates. New J. Phys. 2014, 16, 113067. [CrossRef]

64. Andrews, D.L.; Thirunamachandran, T. On Three-Dimensional Rotational Averages. J. Chem. Phys. 1977, 67, 5026-5033. [CrossRef]

65. Power, E.A.; Thirunamachandran, T. Quantum Electrodynamics with Non-Relativistic Sources. II. Maxwell Fields in the Vicinity of a Molecule. Phys. Rev. A 1983, 28, 2663-2670. [CrossRef]

66. Salam, A. Molecular Quantum Electrodynamics in the Heisenberg Picture: A Field Theoretic Viewpoint. Int. Rev. Phys. Chem. 2008, 27, 405-448. [CrossRef]

67. Agranovitch, M.; Maradudin, A.A. Electronic Excitation Energy Transfer in Condensed Matter; North-Holland: Amsterdam, The Netherlands, 1982.

68. Knoester, J.; Mukamel, S. Intermolecular Forces, Spontaneous Emission, and Superradiance in a Dielectric Medium: Polariton-Mediated Interactions. Phys. Rev. A 1989, 40, 7065-7080. [CrossRef]

69. Juzeliunas, G.; Andrews, D.L. Quantum Electrodynamics of Resonant Energy Transfer in Condensed Matter. Phys. Rev. B 1994, 49, 8751-8763. [CrossRef]

70. Lock, M.P.E.; Andrews, D.L.; Jones, G.A. On the Nature of Long-Range Electronic Coupling in a Medium: Distance and Orientational Dependence for Chromphores in Molecular Aggregates. J. Chem. Phys. 2014, 140, 044103. [CrossRef] [PubMed]

71. Craig, D.P.; Thirunamachandran, T. Chiral Discrimination in Molecular Excitation Transfer. J. Chem. Phys. 1998, 109, 1259-1263. [CrossRef]

72. Salam, A. Resonant Transfer of Excitation Between Two Molecules Using Maxwell Fields. J. Chem. Phys. 2005, 122, 044113. [CrossRef] [PubMed]

73. Scholes, G.D.; Andrews, D.L. Damping and Higher Multipole Effects in the Quantum Electrodynamical Model for Electronic Energy Transfer in the Condensed Phase. J. Chem. Phys. 1997, 107, 5374-5384. [CrossRef] 
74. Salam, A. A General Formula for the Rate of Resonant Energy Transfer Between Two Electric Multipoles of Arbitrary Order Using Molecular Quantum Electrodynamics. J. Chem. Phys. 2005, 122, 044112. [CrossRef] [PubMed]

75. Daniels, G.J.; Andrews, D.L. The Electronic Influence of a Third Body on Resonance Energy Transfer. J. Chem. Phys. 2002, 116, 6701-6712. [CrossRef]

76. Salam, A. Mediation of Resonance Energy Transfer by a Third Molecule. J. Chem. Phys. 2012, 136, 014509. [CrossRef] [PubMed]

77. Andrews, D.L.; Ford, J.S. Resonance Energy Transfer: Influence of Neighbouring Matter Absorbing in the Wavelength Region of the Acceptor. J. Chem. Phys. 2013, 139, 014107. [CrossRef] [PubMed]

78. Ford, J.S.; Andrews, D.L. Geometrical Effects on Resonance Energy Transfer Between Orthogonally-Oriented Chromophores, Mediated by a Nearby Polarisable Molecule. Chem. Phys. Lett. 2014, 591, 88-92. [CrossRef]

79. Salam, A. Long-Range Interparticle Interactions: Insights from Molecular Quantum Electrodynamics (QED) Theory. In Reviews in Computational Chemistry; Parrill, A.L., Lipkowitz, K.B., Eds.; John Wiley \& Sons, Inc.: Hoboken, NJ, USA, 2016; Volume 23, pp. 98-151.

80. Nelson, P.C. The Role of Quantum Decoherence in FRET. Biophys. J. 2018, 114, 167-172. [CrossRef] [PubMed]

(C) 2018 by the author. Licensee MDPI, Basel, Switzerland. This article is an open access article distributed under the terms and conditions of the Creative Commons Attribution (CC BY) license (http:/ / creativecommons.org/licenses/by/4.0/). 